\title{
Centrally acting antihypertensive agent Ketanserin potential role in delirium management: A review
}

\section{OPEN ACCESS}

Citation: Firas Hasan Bazzari (2017) Centrally acting antihypertensive agent Ketanserin potential role in delirium management: A review Open Science Journal 2(3).

Received: $14^{\text {th }}$ June 2017

Accepted: $2^{\text {nd }}$ August 2017

Published: 20 ${ }^{\text {th }}$ September 2017

Copyright:@ 2016 This is an open access article under the terms of the Creative Commons Attribution License, which permits unrestricted use, distribution, and reproduction in any medium, provided the original author and source are credited.

Funding: The author(s) received no specific funding for this work.

Competing Interests: The author have declared that no competing interests exists.

\section{Firas Hasan Bazzari*}

PhD candidate at Cairo University school of Pharmacy, Department of Pharmacology and Toxicology, Cairo, Egypt.

*Corresponding Author: Firas Hasan Bazzari: firas_hasan@rocketmail.com

\section{Abstract}

Delirium is a psychiatric disorder that is well known for being a clinical challenge due to its complex pathophysiology and the wide range of risk factors associated with it. Up to this date, no medical agent is approved by the Food and Drug Administration (FDA) for the treatment of delirium; despite the fact that, a number of clinical guidelines and protocols have recommended the use of some drugs in the management of delirium. Delirium risk factors; predisposing, hospital and acute underlying disease factors, are considered critical points in understanding delirium mechanism. Furthermore, several pathophysiological hypotheses; neurotransmitters imbalance hypothesis, inflammatory hypothesis and structural defects hypothesis, were introduced to explain delirium pathogenesis. Nevertheless, the current evidence is mainly based on and validates the neurotransmitters imbalance theory, since neurotransmitters form the molecular basis of the central nervous system and can be affected by any change occurs in the brain normal conditions. Moreover, pharmacological options introduced as delirium therapies with relative efficacy are mainly based on the neurotransmitters imbalance theory. Ketanserin is a centrally acting antihypertensive agent that has been in the market for over 30 years from now; thus, safety profile is well established. Ketanserin special and unique mechanism of action by inhibiting a wide range of central nervous system receptors; serotonergic (5-HT1D, 5HT2B, 5-HT2C, 5-HT6), dopaminergic (D1, D2) and adrenergic $(\alpha 1, \alpha 2)$ receptors, theoretically seems to match with the pathophysiological hypothesis of neurotransmitters imbalance. Moreover, ketanserin might also serve as an empirical therapy for delirium, when the cause of delirium; other than the underlying medical condition, is not determined and an immediate intervention is needed. As it antagonizes almost all the receptors thought to be associated with delirium. Nevertheless, up to this point, it is still a theoretical assumption about the potential role of ketanserin as a pharmacological option in delirium management that needs further investigations to confirm its efficacy. 
Keywords: Delirium, Ketanserin, Management, Treatments

\section{Introduction}

Delirium is defined as a transient state of confusion that impairs individuals' normal cognitive functions [1]. It could be classified as a syndrome rather than a mental disorder, as it is characterized by a number of defined signs and symptoms associated with its occurrence. For instance, hyper/hypoactivity, inability to focus, illusions, sleep disturbances, perceptual impairments and other cognitive defects [2]. Delirium episodes usually occur due to an organic cause that changes the brain normal conditions, such as an underlying medical condition or a drug-induced case [3]. Therefore, treating the primary cause is the first step in delirium management [4]. Nevertheless, delirium symptoms and diagnosis are frequently confused with other mental disorders [5]; thus, close monitoring and assessment should be undertaken to avoid any possible misdiagnosis.

Elderly hospitalized patients are identified to be at a higher risk of developing delirium episodes [6]. Hospital procedures and hospital environment are known add risk factors other than the underlying condition; for example, ICU (Intensive Care Unit) patients on mechanical ventilation were observed to be $2-3$ times more likely to develop delirium in the ICU compared to non-mechanically ventilated patients [7]. Moreover, delirium episodes could increase the hospitalization period resulting in an increased treatment costs, long-term cognitive impairments and elevated mortality rates within the first year after discharge [8]. Thus, delirium cases should be carefully handled in order to minimize its detrimental futuristic complications.

Pharmacological options, which are the central core of any disease management protocol, are yet to be approved by the FDA for the treatment of delirium [9]. On the other hand, a number of guidelines have suggested the effectiveness and recommended the use of some medical agents, such as typical antipsychotics, atypical antipsychotics, dexmedetomidine and benzodiazepines [10]. Although these pharmacological options seem to be effective and relatively safe as reported by the clinical investigations [11], the pathophysiology of delirium is very complex and the disease molecular mechanism is not fully understood. Thus, more studies should be undertaken in order to approve the use of the current pharmacological therapies.

Ketanserin is a centrally acting antihypertensive agent that was discovered in 1980, which is clinically used in patients with hypertension; in addition to, its use in understanding the serotonin system in scientific research [12]. The theoretical assumption about ketanserin potential role in delirium treatment is mainly based on its unique mechanism of action that might fit with the introduced pathophysiological hypotheses of delirium. Therefore, the pathophysiological hypotheses and delirium risk factors would be explained in order to assess the relation between ketanserin and delirium mechanism. 


\section{Delirium risk factors}

The risk factors of delirium can be classified into three major categories; predisposing, hospital related and the underlying acute condition factors. Moreover, multiple risk factors could be added together and elevate the risk and severity of delirium, such as the case of hospitalized ICU patients who may have over ten different risk factors at the same time [13].

\section{Predisposing factors (Baseline factors)}

Predisposing factors are commonly related to the individual's normal life habits or medical history. These risk factors may include; patient's age [14], smoking [15], alcohol consumption [16], history of depression [17], history of hypertension [18] and other factors related to genetic disposition, such as APOE4 (Apolipoprotein - 4) polymorphism [19]. However, those risk factors are critical and should be assessed and taken into consideration prior the start of the medical treatment or hospital admission in order to minimize the risk of developing delirium.

\section{Hospital related factors}

For patients who require hospitalization, risk factors are mainly related to the surrounding hospital environment. For example, physical restrain and patient immobility are suggested to increase delirium risk [20]. Moreover, sleep deprivation and sleep disturbances, which frequently occur in hospitalized patients, are major contributors in precipitating delirium [20].

\section{Underlying acute condition risk factors}

The underlying acute condition is the main identified cause of delirium. However, many factors could be related to its severity and the type of medical intervention used. These factors might include; the use of mechanical ventilation, surgical operations, polypharmacy, metabolic disturbances, elevated levels of inflammatory biomarkers and sepsis development [21].

\section{Delirium pathophysiology hypotheses}

A number of pathophysiological hypotheses were introduced in order to figure out delirium mechanism. The hypotheses are; neurotransmitters imbalance hypothesis, inflammatory hypothesis and structural defects hypothesis. These hypotheses are still theories that are yet to be approved; however, clinical trials to some extent have generated an evidence that suggests their viability in explaining delirium pathogenesis.

\section{Neurotransmitters imbalance hypothesis}

Neurotransmitters play a vital role in cell signaling that controls almost all physiological functions. Disturbance in the levels of neurotransmitters is thought to be one of the major contributors in delirium pathogenesis. Imbalanced levels 
of; acetylcholine, dopamine, serotonin, GABA (Gamma Amino Butyric Acid) and glutamate, are suggested to participate in precipitating delirium.

\section{Acetylcholine}

Cholinergic deficiency theory is suggested to be strongly associated with delirium development. In which, studies have compared levels of acetylcholine with the incidence of delirium and found that delirium occurs more frequently in patients with low levels of acetylcholine [22]. Moreover, the reticular activating system in the central nervous system, which is responsible for maintaining the circadian rhythm (sleep cycle), is also suggested to have a role in reducing acetylcholine levels in the brain due to sleep disturbances [23]. Thus, explaining the increased delirium incidence in hospitalized patients who often have sleep issues.

\section{Dopamine}

Dopamine is another major neurotransmitter in the central nervous system. However, it is found to have an inverse relation with acetylcholine; in other words, patients with elevated levels of dopamine have lowered levels of acetylcholine and vice versa [24]. This could be due to the existence of dopamine receptors - D2 subtype near the cholinergic fibers in the brain cortex [25]. Thus, resulting in reducing acetylcholine synthesis and cholinergic deficiency. Moreover, increased levels of dopamine are associated with psychotic symptoms and blurred thinking, which are both signs of delirium [25].

\section{Serotonin}

Serotonin neurotransmitter synthesis is quite different from other neurotransmitters, as serotonin is mainly derived from the amino acid tryptophan, while the other majority of neurotransmitter are derived from tyrosine [26]. However, scientists have developed a measuring method tryptophan/LNAA (Large Neutral Amino Acids) ratio to measure serotonin levels, since tryptophan crosses the blood-brain barrier via the aid of specific transporters that usually competes with LNAA [26]. However, delirium incidence could be as a result of both increased and reduced levels of serotonin. For example, in the case of hepatic encephalopathy, which attenuates the blood-brain barrier, serotonin synthesis will increase; thus, developing psychotic symptoms and elevating the risk of developing serotonin syndrome [27]. On the other hand, reducing the blood-brain barrier permeability would result in reducing serotonin levels; for instance, delirium associated with patients receiving levodopa and in the case of alcohol withdrawal [26]. Moreover, disturbed levels of serotonin are suggested to lower cholinergic activity; in addition to, increased levels of serotonin are found to promote dopamine release [28].

\section{Norepinephrine}

Norepinephrine is an adrenergic neurotransmitter that is identified to have various biological functions in the central nervous system; for instance, neuroplasticity, cell metabolism, regulation of cortical circuits and inflammation [29]. It has also found to play a role in attention, controlling the mood and anxiety, which is quite similar to dopamine [29]. Furthermore, in delirium 
episodes increased levels of norepinephrine has two main related modes of action; increasing dopamine activity and lowering acetylcholine levels, which are both considered hallmarks of delirium pathophysiology [28].

\section{GABA and glutamate}

Despite the fact that glutamate is the precursor of GABA; nevertheless, they both identified to play totally different functions. In which, glutamate is the main excitatory neurotransmitter in the central nervous system, while GABA is the major inhibitory neurotransmitter [30]. Their mechanism in precipitating delirium is not fully understood; however, it is suggested to have a relation with cholinergic deficiency [28]. For example, low levels of GABA in the case of sedatives or hypnotics withdrawal are observed to induce delirium; thus, suggesting that lowered levels of GABA are associated with delirium development [31].

\section{Inflammatory hypothesis}

This theory suggests the involvement of a number of immune system components in delirium pathogenesis. In the case of hospitalized patients, there is a higher risk of acquiring an infection from the hospital environment, that may aggravate to a generalized infection [32]. A generalized infection exposes the human body to a massive amount of immune system components [32]. For instance, clinical studies have shown a link between increased levels of IL-6 (Interleukin-6), IL-8 (Interlaken-8) and delirium in elderly individuals [33]. However, the exact mechanism by which they induce delirium is still unclear [33].

\section{Structural defects hypothesis}

The structural defects and anatomical changes are major factors in the disruption of the central nervous system homeostasis. Therefore, it is believed to have a role in the pathogenesis of delirium. For example, patients with strokes have a higher risk of developing delirium [34]; moreover, COMT (catechol-Omethyltransferase) gene polymorphism in alcohol withdrawal associated delirium [35]. Furthermore, blood-brain barrier defect is another vital player in exposing the central nervous system to a massive range of neurotoxins that would participate in delirium development [36]. Thus, identifying those structural defects might significantly reduce the occurrence of delirium.

According to the three major previously mentioned pathophysiological theories of delirium, the neurotransmitters imbalance theory seems to be the most viable one. This assumption is made based on the fact that neurotransmitters are the smallest molecular level of any of the body systems; therefore, other theories regarding inflammation and structural defects may to some extent have an effect on the neurotransmitters of the central nervous system. Moreover, the current pharmacological options that follow the neurotransmitters theory seems to be effective and relatively safe. 


\section{Ketanserin potential role}

According to the early mentioned points, it could be suggested that targeting the neurotransmitters systems might have positive outcomes in treating delirium or preventing it in the first place, especially in the individuals who have multiple risk factors. There are few reasons based on a scientific evidence about the theoretical assumption made on the potential role of ketanserin in delirium. Firstly, ketanserin is already a drug in use in patients with hypertension for decades; therefore, it is an available pharmacological agent that already passed human safety assessments in clinical trials [37]. Moreover, there are no reported cases of increased delirium risk in patients receiving ketanserin compared to other centrally acting antihypertensive; for example, clonidine that is identified to be associated with increased risk of delirium [38].

Furthermore, the most vital point about ketanserin is its mechanism of action, which is characterized by a wide range of antagonistic activity towards many subtypes of central nervous system receptors. Ketanserin is found to have a high antagonistic activity on the serotonin receptor subtype 5HT2A and H1 histamine receptor [12]. Moreover, at lower affinity ketanserin is also found to antagonize; serotonin receptors subtypes (5-HT1D, 5-HT2B, 5-HT2C, 5-HT6), dopamine receptors (D1, D2) and adrenergic receptors $(\alpha 1, \alpha 2)$ [12]. According to the neurotransmitters imbalance theory, it could be observed that there is a relation between the mechanism of action of ketanserin and delirium pathogenesis. As ketanserin could be able to lower levels of dopamine, serotonin and norepinephrine, in which their elevated levels are associated with precipitating delirium and psychotic symptoms [39]. Moreover, ketanserin could also indirectly increase acetylcholine due to the inhibition of its suppressors; dopamine, serotonin and norepinephrine [28]. Therefore, ketanserin unique mechanism of action theoretically matches the neurotransmitters imbalance theory of delirium.

Regarding the ability of ketanserin to block a huge number of receptors, it could be argued that it may potentially increase unwanted adverse events. Nevertheless, clinical investigations made on various pharmacological agents have shown that these drugs are effective in treating delirium at low doses with minimal side effects [40]. For instance, a study on haloperidol, which is considered the current gold standard treatment of delirium by blocking the dopamine-D2 receptor, has shown a lowered risk of developing extrapyramidal side effects associated with the use of haloperidol [41]. In addition, delirium is an acute and transient disorder; in other words, the patient exposure to the treatment would be for a very short period of time (hours/days) [42]. Thus, the risk of the drug to be build up in the body would be reduced [41]. Lastly, ketanserin antagonizes all receptors that are thought to be associated with delirium; thus, it could serve as an empirical therapeutic agent in the case of the unknown cause of delirium episodes, whether it is due to an increase in serotonin, norepinephrine or dopamine levels.

Ultimately, ketanserin appears to have a potential role in delirium management according to its mechanism of action and the disease pathogenesis. However, it should be pointed out that it is only theoretical assumptions based on the scientific evidence available about delirium and ketanserin. Therefore, 
further clinical investigations are needed to confirm the efficacy and activity of ketanserin in the treatment process of delirium.

\section{Conclusion}

In conclusion, delirium is a serious medical issue that has massive negative complications that significantly lowers the patients' quality of life. Individuals may enter the hospital for an acute medical issue and discharge with a lifetime cognitive impairments because of delirium. Thus, aiming to find and discover novel pharmacological options for the treatment of delirium is a current major trend that has been gaining momentum in the past years. According to the current pathophysiology of delirium, ketanserin mode of action could possibly benefit in delirium management. Furthermore, Ketanserin seems as a feasible, more practical and cost-effective option, since it has already been clinically tested and approved as an antihypertensive; hence, it has an established safety profile. Nevertheless, ketanserin has never been tested on delirium patients to assess its efficacy and it is still a theoretical assumption based on current literature evidence. Therefore, it is well recommended to undertake further investigations to examine its actual potential.

\section{References}

Lipowski Z. Delirium (acute confusional states). JAMA. 1987; 258(13): p. 1789-1792.

Stango D, Gibson C, Breitbart W. The delirium subtypes: a review of prevalence, phenomenology, pathophysiology, and treatment response. Palliative \& supportive care. 2004; 2(2): p. 171-179.

Rockwood K. Causes of delirium. Psychiatry. 2008; 7(1): p. 39-41.

Rudolph JL, Archambault E, Budd MA. Delirium: Risk Identification, Mitigation, and Intervention. Practical Psychology in Medical Rehabilitation. 2017;: p. 235-239.

Otani VHO, Otani TZDS, Freirias A, Calfat ELDB, Aoki PS, Cordeiro Q, et al. Misidentification of mental health symptoms in presence of organic diseases and delirium during psychiatric liaison consulting. nternational Journal of Psychiatry in Clinical Practice. 2017;: p. 1-6.

Anand A, MacLullich AM. Delirium in hospitalized older adults. Medicine. 2017; 15(1): p. 46-50.

Brummel N, Girard T. Preventing delirium in the intensive care unit. Critical care clinics. 2013; 29(1): p. 51-65.

Fong T, Tulebaev S, Inouye S. Delirium in elderly adults: diagnosis, prevention and treatment. Nature Reviews Neurology. 2009; 5(4): p. 210-220.

FDA. FDA. [Online].; 2017 [cited 2017 March 20. Available from: HYPERLINK "https://www.fda.gov/Drugs/default.htm" https://www.fda.gov/Drugs/default.htm .

Fosnight S. DELIRIUM IN THE ELDERLY. Geriatrics. 2011;: p. 73-96.

Barr J, Fraser GL, Puntillo K, Ely EW, Gélinas C, Dasta JF, et al. Clinical practice guidelines for the management of pain, agitation, and delirium in adult patients in the intensive care unit. Critical Care Medicine. 2013; 41(1): p. 263-306.

Olivier B, Van Wijngaarden I, Soudijn W. Serotonin receptors and their ligands. Elsevier. 1997; 27.

Fleet J, Ernst T. Guy's and St Thomas. [Online].; 2011 [cited 2017 April 2. Available from: HYPERLINK "http://www.guysandstthomas.nhs.uk/resources/our-services/acute-medicine-gisurgery/elderly-care/delirium-adult-inpatients.pdf" http://www.guysandstthomas.nhs.uk/resources/our-services/acute-medicine-gi-surgery/elderlycare/delirium-adult-inpatients.pdf .

McNicoll L, Pisani MA, Zhang Y, Ely E, Siegel MD, Inouye SK. Delirium in the intensive care unit: occurrence and clinical course in older patients. Journal of the American Geriatrics Society. 2003; 51(5): p. 591-598. 
Hsieh SJ, Shum M, Lee AN, Hasselmark F, Gong MN. Cigarette smoking as a risk factor for delirium in hospitalized and intensive care unit patients. A systematic review. Annals of the American Thoracic Society. 2013; 10(5): p. 496-503.

Monte R, Rabuñal R, Casariego E, Bal M, Pértega S. Risk factors for delirium tremens in patients with alcohol withdrawal syndrome in a hospital setting. European journal of internal medicine. 2009; 20(7): p. 690-694.

Tully P, Baker R, Winefield H, Turnbull D. Depression, anxiety disorders and Type D personality as risk factors for delirium after cardiac surgery. Australian and New Zealand Journal of Psychiatry. 2010; 44(11): p. 1005-1011.

Ouimet S, Kavanagh B, Gottfried S, Skrobik Y. Incidence, risk factors and consequences of ICU delirium. Intensive Care Medicine. 2007; 33(1): p. 66-73.

Canonico A, Light R, Pandharipande P, Laskowitz D. Apolipoprotein E4 polymorphism as a genetic predisposition to delirium in critically ill patients. Critical Care Medicine. 2007; 119(6): p. 737754.

McCusker J, Cole M, Abrahamowicz M, Han L, Podoba JE, Ramman - Haddad L. Environmental risk factors for delirium in hospitalized older people. Journal of the American Geriatrics Society. 2001; 49(10): p. 1327-1334.

Inouye S. Delirium in hospitalized older patients. Clinics in Geriatric Medicine. 1998; 14(4): p. 749764.

Tune L, Egeli S. Acetylcholine and delirium. Dementia and geriatric cognitive disorders. 1999; 10(5): p. $342-344$

Ross C. CNS arousal systems: possible role in delirium. International Psychogeriatrics. 1991; 3(2): p. 353-371.

KIM JY, JUNG IK, Han C, CHO SH, Kim L, KIM SH, et al. Antipsychotics and dopamine transporter gene polymorphisms in delirium patients. Psychiatry and clinical neurosciences. 2005; 59(2): p. 183-188.

Mrzljak L, Goldman - Rakic P. Acetylcholinesterase reactivity in the frontal cortex of human and monkey: Contribution of AChE - rich pyramidal neurons. Journal of Comparative Neurology. 1992; 324(2): p. 261-281.

Van Der Mast R, Fekkes D. Serotonin and amino acids: partners in delirium pathophysiology? Seminars in clinical neuropsychiatry. 2000; 5(2): p. 125-131.

Knell AJ, Davidson AR, Williams R, Kantamaneni BD, Curzon G. Dopamine and serotonin metabolism in hepatic encephalopathy. Br Med J. 1974; 1(5907): p. 549-551.

Hshieh T, Fong TG, Marcantonio ER, Inouye S. Cholinergic Deficiency Hypothesis in Delirium: A Synthesis of Current Evidence. The Journals of Gerontology. 2008; 63(7): p. 764-772.

O'Donnell J, Zeppenfeld D, McConnell E, Pena S, Nedergaard M. Norepinephrine: a neuromodulator that boosts the function of multiple cell types to optimize CNS performance. Neurochemical research. 2012; 37(11): p. 2496-2512.

Petroff O. GABA and glutamate in the human brain. The Neuroscientist. 2002; 8(6): p. 562-573.

Ali S, Patel M, Jabeen S, Bailey R, Patel T, Shahid M. Insight into Delirium. Innovations in Clinical Neuroscience. 2011; 8(10): p. 25-34.

Ebersoldt M, Sharshar T, Annane D. Sepsis-associated delirium. Intensive care medicine. 2007; 33(6): p. 941-950.

Van Munster BC, Korevaar JC, Zwinderman AH, Levi M, Wiersinga WJ, De Rooij SE. Time - course of cytokines during delirium in elderly patients with hip fractures. Journal of the American Geriatrics Society. 2008; 56(9): p. 1704-1709.

Soiza RL, Sharma V, Ferguson K, Shenkin SD, Seymour DG, MacLullich AM. Neuroimaging studies of delirium: a systematic review. Journal of psychosomatic research. 2008; 65(3): p. 239-248.

Nakamura A, Inada T, Kitao Y, Katayama Y. Association between catechol - O - methyltransferase (COMT) polymorphism and severe alcoholic withdrawal symptoms in male Japanese alcoholics. Addiction biology. 2001; 6(3): p. 233-238.

Davies D. Blood-brain barrier breakdown in septic encephalopathy and brain tumours. Journal of anatomy. 2002; 200(6): p. 639-646.

Mauersberger H. Long-term safety and efficacy of ketanserin in essential hypertension: ketanserin versus or in combination with metoprolol. Journal of hypertension. 1986; 4(1): p. 73-75.

Delaney J, Spevack D, Doddamani S, Ostfeld R. Clonidine-induced delirium. International journal of cardiology. 2006; 113(2): p. 276-278.

Richtand NM, Welge JA, Logue AD, Keck PE, Strakowski SM, McNamara RK. Dopamine and serotonin receptor binding and antipsychotic efficacy. Neuropsychopharmacology. 2007; 32(8): p. $1715-1726$. 
Sasaki Y, Matsuyama T, Inoue S, Sunami T, Inoue T, Denda K, et al. A prospective, open-label, flexible-dose study of quetiapine in the treatment of delirium. Journal of Clinical Psychiatry. 2003; 64(11): p. 1316-1321.

Meagher DJ. Regular review: Delirium: optimising management. BMJ: British Medical Journal. 2001; 322(7279): p. 144 .

Rockwood K. The occurrence and duration of symptoms in elderly patients with delirium. Journal of Gerontology. 1993; 48(4): p. 162-166.Social Sciences Conference in Kolkata-India. 18-20 December 2015 